\title{
Rate-Adaptive Information Transmission over MIMO Channels
}

\author{
Marco Zoffoli, Jerry D. Gibson and Marco Chiani \\ Fellow, IEEE
}

\section{Introduction}

In the context of wireless communication, a Multiple-Input Multiple-Output (MIMO) system is a system that employs multiple antennas both at the transmitter and receiver. The first theoretical analysis of MIMO systems were developed by Winters (1987), Foschini (1996) and Telatar (1999), and since then there have been many research efforts on this subject. What mainly makes MIMO systems interesting is their potential ability to achieve an increase in system capacity or in link reliability without requiring additional transmission power or bandwidth (Goldsmith, 2005).

In this work, we focus on the utilization of MIMO systems for the lossy transmission of source information. In particular, we want to compare several different strategies for the transmission of a zero mean Gaussian source over Rayleigh-fading MIMO channels, assuming rate-adaptive source encoding. The MIMO transmission strategies are based on techniques such as Repetition coding (REP), Time Sharing (TS), the Alamouti scheme (ALM) and Spatial Multiplexing (SM) (Alamouti, 1998; Tse \& Viswanath, 2006).

Depending on its characteristics, each strategy will be used either for the transmission of a Single Description (SD) or the transmission of a Multiple Description (MD) representation of this source. In SD coding, a single stream of information describing the source is transmitted over a single channel. In MD coding (Gamal \& Cover, 1982), the source is represented using two different descriptions that are transmitted over two independent channels. If both descriptions are correctly received, they can be combined together at the receiver to obtain a reconstruction of the source at a certain quality. If only one of the two descriptions is correctly received, a reconstruction of the source is still possible but at a lower quality.

We consider adaptive source encoding, where the rate is adapted to follow the slow variations of the channel (due e.g. to shadowing and path loss) or the fast variations of the channel (due to fading), leading to two scenarios that we call fixed-outage and zero-outage, respectively. In the first case, we consider Gaussian source transmission over MIMO systems when CSI is not available at the transmitter. In this scenario, since the transmitter does not have knowledge of channel state information (CSI), it does not know the instantaneous rate supported by the channel, i.e. its capacity, and hence it is not able to adapt the source coding rate to the channel conditions to ensure the decoding of the information at the receiver with an arbitrarily small probability of error. Instead, it encodes and transmits the source information using a rate chosen to achieve a selected outage probability. When the channel does not support the 
transmission of information at the chosen coding rate, data are lost and the system experiences an outage. We call this the fixed-outage rate-adaptive approach.

In the second scenario (zero-outage rate-adaptive), we consider the different MIMO strategies under the assumption of perfect CSI at the transmitter. In this case, the transmitter is able to follow the variations of the channel by adapting the source coding rate to the instantaneous capacity, since it is aware of the particular channel realization in every time instant. In such a situation there is no outage since the source rate is always adapted to achieve the instantaneous channel capacity (Choudhury \& Gibson, 2007). This observation has a direct impact on the usefulness of the TS strategies in the zero-outage scenario. These strategies employ a time sharing approach to the transmit antennas to create independent channels from our MIMO system (Zoffoli et al., 2008a). These independent channels are then used to provide path diversity by transmitting multiple description representations of the source over them. However, path diversity is useful only if the channels are unreliable, i.e. if they suffer outages. For this reason, in the zero-outage scenario we do not consider the TS strategies.

The different strategies for both the fixed-outage and the zero-outage rate adaptation approaches are described in the following sections, where we also evaluate their performance by studying the statistics of the distortion at the receiver.

In the presence of outage, it is usually assumed either implicitly or explicitly that retransmissions will be used for data scheduled to be transmitted during an outage; indeed, choosing an operational outage rate may be associated with an acceptable retransmission rate. Although retransmissions are the natural response to outage for data sources, relying on retransmissions may or may not be appropriate for compressed voice or video for several reasons. First, it is not unusual to rely on packet loss concealment for voice and video up to some non-trivial packet loss rate. Second, it may be more desirable not to retransmit for voice and video in order to reduce latency or to maximize access point throughput. As a result, the suitable measure of performance for lossy source coding of voice and video is the average distortion of the source reproduced at the receiver. Average distortion is also the appropriate performance indicator for the zero outage rate case, since we are adapting the source coding rate to the instantaneous capacity of the channel, and it is desired to determine the reproduced quality of the source delivered to the user. Therefore, for our work here, we choose the mean squared error (MSE) fidelity criterion.

In Section II, we present the basic assumptions and set up the particular MIMO problems we are addressing. Section III contains the development of the fixed outage rate adaptive source encoding scenarios we examine, including the repetition strategy and single description source coding, the time sharing strategy and the three multiple descriptions source coding methods (no excess marginal rate, no excess joint rate, and optimized multiple descriptions source coding), the Alamouti strategy with single description source coding, and spatial multiplexing with single description source coding. Zero outage rate adaptive source encoding, wherein CSI is available at the transmitter and the source coding is adjusted to match the instantaneous capacity, is described in Section IV, including the developments and derivations of the distributions of the reconstructed source distortion for the repetition, Alamouti, and spatial multiplexing strategies. Extensive results for each of the methods and comparisons of the results are presented in Section V, while Section VI summarizes the conclusions from the work. 


\section{Assumptions and preliminaries}

Our main goal is to discuss how MIMO techniques impact on adaptive source encoding. Although most of our results can be easily extended to cover the general $N_{t} \times N_{r}$ MIMO channel case, for the sake of simplicity we consider the frequency-flat $2 \times 2$ MIMO channel. The system is characterized by the channel matrix $H$, having the form

$$
H=\left(\begin{array}{ll}
h_{11} & h_{12} \\
h_{21} & h_{22}
\end{array}\right)
$$

Each entry $h_{i j}$ of the channel matrix $H$ represents the gain of the channel between the $j$-th transmit antenna and $i$-th receive antenna. These channels are assumed to be independent, random and with very slow Rayleigh fading. The $h_{i j}$ are then i.i.d. complex Gaussian random variables with zero mean and unit variance, which remain constant over the transmission of a large number of symbols. Under these assumptions, the squared magnitude of the channel gains can be written as

$$
\left|h_{i j}\right|^{2}=\frac{1}{2} x_{i j}, \quad i, j=1,2
$$

where the $x_{i j}$ are random variables distributed according to a chi-square distribution with 2 degrees of freedom (Hogg \& Craig, 1970). Perfect CSI, i.e. knowledge of $H$, is assumed to be always available at the receiver, while the transmitter has a full or partial CSI depending on the scenario, as will be discussed later.

The total transmitted power by the transmit antennas is constrained to $P_{t}$. If both transmit antennas are transmitting simultaneously, each antenna will transmit with equal power $P_{t} / 2$, while, if only one antenna is transmitting at a given time, it can make use of full transmit power $P_{t}$. The noise at the receiver is AWGN, with i.i.d. statistics and the same average power $N$ at each receive antenna.

We denote with $\gamma_{i j}$ the instantaneous Signal to Noise Ratio (SNR) of the signal transmitted by the $j$-th antenna and received by the $i$-th antenna. Thus,

$$
\gamma_{i j}=\frac{P_{t}}{N}\left|h_{i j}\right|^{2}=\bar{\gamma}\left|h_{i j}\right|^{2}, \quad i=1,2
$$

if only the $j$-th antenna is transmitting at a given time, and

$$
\gamma_{i j}=\frac{P_{t}}{2 N}\left|h_{i j}\right|^{2}=\frac{\bar{\gamma}}{2}\left|h_{i j}\right|^{2}, \quad i, j=1,2
$$

if both antennas are transmitting at the same time.

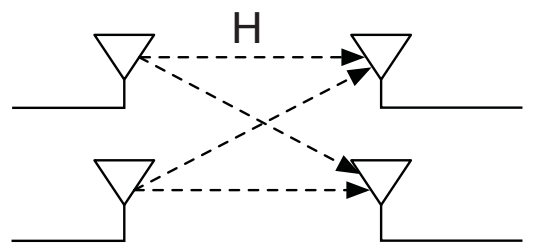

Fig. 1. $2 \times 2 \mathrm{MIMO}$ model.

The source is assumed to be a zero-mean memoryless Gaussian source with a variance normalized to unity. The system bandwidth is also assumed to be normalized to unity. 
In the following, we will denote with $\bar{\gamma}$ the ratio $P_{t} / N$ and with $\Gamma(z)$ and $\Gamma(a, z)$, respectively, the gamma function and the incomplete gamma function (Hogg \& Craig, 1970). We will also denote with $\chi_{k}^{2}$ the distribution of a chi-square random variable with $k$ degrees of freedom, with $F_{\chi}^{(k)}(z)$ its CDF and with

$$
f_{\chi}^{(k)}(z)=\frac{1}{\Gamma\left(\frac{k}{2}\right) 2^{\frac{k}{2}}} z^{\frac{k-2}{2}} e^{-\frac{z}{2}}
$$

its probability density function (PDF) (Hogg \& Craig, 1970).

\section{Fixed-Outage rate-adaptive source encoding (FORA)}

In a wireless channel, due to multipath propagation and users' mobility, the capacity is varying in time. In this section we assume that the source encoder knows only the statistical distribution of the wireless channel mutual information, and that it adapts its rate accordingly. The source encoder rate is chosen to produce a certain outage probability, determined to minimize the distortion at the received end. This could be assumed a slow-adaptive technique, since the source encoder rate will follow the variations of the channel statistics due, for instance, to shadowing and path loss changes.

\subsection{Repetition}

The REP strategy is based on repetition coding (Tse \& Viswanath, 2006). The basic idea is to transmit the same symbol over the two transmit antennas in two consecutive time slots. In each time slot, only one of the two transmit antennas is used for transmission, while the other antenna is turned off.

Thus, in the first time slot the symbol $S_{1}$ is transmitted on the first transmit antenna and it is observed by the receiver through the two channels with gains $h_{11}$ and $h_{21}$. In the second time slot, the same symbol $S_{1}$ is transmitted on the second transmit antenna and it is observed by the receiver through the two channels with gains $h_{12}$ and $h_{22}$. A Maximal Ratio Combiner (MRC) (Goldsmith, 2005) is then used at the receiver to optimally combine the four signals received by the two receive antennas in the two different time slots.

The instantaneous SNR $\gamma$ of the signal at the output of the MRC is given by the sum of the instantaneous SNRs $\gamma_{i j}$ of its input signals (Goldsmith, 2005), that are given by Eq. (2)

$$
\gamma=\sum_{i, j=1}^{2} \gamma_{i j}=\bar{\gamma} \sum_{i, j=1}^{2}\left|h_{i j}\right|^{2}
$$

In this way, a single channel is obtained from the four independent channels available in our MIMO system. This strategy is then suitable for the transmission of a SD representation of the source.

The instantaneous capacity of this single channel in [bits/channel use] is given by (Goldsmith, 2005)

$$
C=\frac{1}{2} \log _{2}(1+\gamma)=\frac{1}{2} \log _{2}\left(1+\bar{\gamma} \sum_{i, j=1}^{2}\left|h_{i j}\right|^{2}\right)
$$

where the factor $1 / 2$ arises because we are transmitting the same symbol over two consecutive time slots. 
The source coding rate $R_{R E P}$ of the SD coder is chosen to be equal to the outage capacity at a given value for the outage probability $P_{\text {out }}$, i.e. it is chosen such that

$$
\operatorname{Pr}\left\{C<R_{R E P}\right\}=P_{\text {out }}
$$

Thus, with probability $1-P_{\text {out }}$ the system is not in outage, which means that it can support the transmission at a rate $R_{R E P}$ with an arbitrarily small probability of error, since its capacity is higher than $R_{R E P}$ (Cover \& Thomas, 1991). In such case, the receiver is able to reconstruct the source information with a distortion $D_{1}$ equal to (Cover \& Thomas, 1991)

$$
D_{1}=2^{-2 R_{R E P}}
$$

If the system results in outage, which happens with probability $P_{\text {out }}$, the receiver is not able to correctly decode the transmitted information with an arbitrarily small probability of error and achieves a distortion equal to 1.

The expected distortion $D$ at the receiver is then

$$
D=\left(1-P_{\text {out }}\right) D_{1}+P_{\text {out }}
$$

The outage rate $R_{R E P}^{\text {out }}$, defined as the average rate correctly received over many transmission bursts (Goldsmith, 2005), is given by

$$
R_{R E P}^{\text {out }}=\left(1-P_{\text {out }}\right) R_{R E P}
$$

\subsection{Time sharing - multiple description (TS-MD)}

In this strategy a TS approach is adopted to obtain two independent channels from the MIMO system. The idea behind this strategy is to transmit two different symbols over the two transmit antennas in two consecutive time slots. In each time slot, only one of the two transmit antennas is used for transmission, while the other antenna is turned off. Thus, in the first time slot the first symbol $S_{1}$ is transmitted over the first antenna and it is observed by the receiver through the two channels with gains $h_{11}$ and $h_{21}$. In the second time slot, the second symbol $S_{2}$ is transmitted over the second antenna and it is observed by the receiver through the two channels with gains $h_{12}$ and $h_{22}$. The receiver will then combine the two signals received in the same time slot using a MRC.

Since each received signal has a SNR given by Eq. (2), the signal at the output of the MRC in the $j$-th time slot has a SNR equal to (Goldsmith, 2005)

$$
\gamma_{j}=\sum_{i=1}^{2} \gamma_{i j}=\bar{\gamma} \sum_{i=1}^{2}\left|h_{i j}\right|^{2}
$$

In this way, two independent channels are effectively created in the two time slots, making this strategy suitable for the transmission of a MD representation of the source.

The channel at the $j$-th time slot has an instantaneous capacity $C_{j}$ equal to (Goldsmith, 2005)

$$
C_{j}=\frac{1}{2} \log _{2}\left(1+\gamma_{j}\right)=\frac{1}{2} \log _{2}\left(1+\bar{\gamma} \sum_{i=1}^{2}\left|h_{i j}\right|^{2}\right)
$$

where the factor $1 / 2$ arises because each channel is used only half of the time. 
The side description rate $R_{M D} / 2$, which equals the transmitted rate over each channel, is chosen to be equal to the outage capacity for a given $P_{\text {out }}$, i.e. is chosen such that

$$
\operatorname{Pr}\left\{C_{j}<\frac{R_{M D}}{2}\right\}=P_{\text {out }}
$$

The expected distortion $D$ at the receiver is then given by

$$
D=\left(1-P_{\text {out }}\right)^{2} D_{0}+2 P_{\text {out }}\left(1-P_{\text {out }}\right) D_{1}+P_{\text {out }}^{2}
$$

where $D_{0}$ and $D_{1}$ are the distortions achieved by the receiver when observing, respectively, both descriptions or only one of the two descriptions.

Depending on the type of MD coder used, $D_{0}$ and $D_{1}$ can have different expressions (Balam \& Gibson, 2006) and different TS-MD strategies can be obtained. The No Excess Marginal Rate coder (MD-NMR) (Balam \& Gibson, 2006) is employed in the TS-MD-NMR strategy. The side descriptions are then rate distortion optimal and the distortions have the following expressions (Balam \& Gibson, 2006; Effros et al., 2004)

$$
\begin{aligned}
& D_{0}=\frac{2^{-R_{M D}}}{2-2^{-R_{M D}}} \\
& D_{1}=2^{-R_{M D}}
\end{aligned}
$$

The No Excess Joint Rate coder (MD-NJR) (Balam \& Gibson, 2006) is employed in the TS-MD-NJR strategy. Here the joint description is rate distortion optimal and the distortions have the following expressions (Balam \& Gibson, 2006; Effros et al., 2004)

$$
\begin{aligned}
& D_{0}=2^{-2 R_{M D}} \\
& D_{1}=\frac{1}{2}\left(1+2^{-2 R_{M D}}\right)
\end{aligned}
$$

The optimal coder (MD-OPT) (Effros et al., 2004) is employed in the TS-MD-OPT strategy. In this case, neither the side descriptions nor the joint description is rate distortion optimal, but they are chosen to minimize the expected distortion $D$ in Eq. (7) for a given $P_{\text {out }}$. The distortions $D_{0}$ and $D_{1}$ are given by the following expression (Balam \& Gibson, 2006; Effros et al., 2004)

$$
\left(D_{0}, D_{1}\right)=\left(a, \frac{1+a}{2}-\frac{1-a}{2} \sqrt{1-\frac{2^{-2 R_{M D}}}{a}}\right)
$$

with

$$
a \in\left[2^{-2 R_{M D}}, \frac{2^{-R_{M D}}}{2-2^{-R_{M D}}}\right]
$$

Thus, the MD-OPT coder chooses the proper value for $a$ to minimize the expected distortion $D$.

The outage rate $R_{M D}^{\text {out }}$ is given by

$$
\begin{aligned}
R_{M D}^{\text {out }} & =\left(1-P_{\text {out }}\right)^{2} R_{M D}+2 P_{\text {out }}\left(1-P_{\text {out }}\right) \frac{R_{M D}}{2} \\
& =\left(1-P_{\text {out }}\right) R_{M D}
\end{aligned}
$$




\subsection{Alamouti}

This strategy employs the Alamouti scheme (Alamouti, 1998; Tse \& Viswanath, 2006) to obtain two independent channels from the MIMO system. Since both channels have the same gain given by $\sum_{i, j=1}^{2}\left|h_{i j}\right|^{2}$ (Tse \& Viswanath, 2006), it is evident that it is impossible to have, for a given realization of the channel matrix $H$, one channel in outage and the other not in outage, i.e. both channels can only be simultaneously in outage or simultaneously not in outage. ${ }^{1}$ This strategy is then not suitable for the transmission of a multiple description representation of the source, as also pointed out in (Effros et al., 2004). Instead, it could be used for the transmission of a single description representation, demultiplexing it into two half-rate substreams which are then transmitted over the two channels.

The signals at the output of the Alamouti decoder have the same instantaneous SNR $\gamma$, equal to the sum of the SNRs of the signals on each branch (Goldsmith, 2005). Thus, from Eq. (3) we have

$$
\gamma=\sum_{i, j=1}^{2} \gamma_{i j}=\frac{\bar{\gamma}}{2} \sum_{i, j=1}^{2}\left|h_{i j}\right|^{2}
$$

The instantaneous capacity of this system is then given by (Sandhu \& Paulraj, 2000)

$$
C=\log _{2}(1+\gamma)=\log _{2}\left(1+\frac{\bar{\gamma}}{2} \sum_{i, j=1}^{2}\left|h_{i j}\right|^{2}\right)
$$

and the source coding rate $R_{A L M}$ is chosen such that

$$
\operatorname{Pr}\left\{C<R_{A L M}\right\}=P_{\text {out }}
$$

The expected distortion is then

$$
D=\left(1-P_{\text {out }}\right) D_{1}+P_{\text {out }}
$$

where $D_{1}$ is the distortion achieved by the receiver when the system is not in outage, which is equal to (Cover \& Thomas, 1991)

$$
D_{1}=2^{-2 R_{A L M}}
$$

The outage rate $R_{A L M}^{\text {out }}$ is given by

$$
R_{A L M}^{\text {out }}=\left(1-P_{\text {out }}\right) R_{A L M}
$$

\subsection{Spatial multiplexing}

In the SM strategy (Tse \& Viswanath, 2006), a single symbol stream is first demultiplexed and encoded into two separate and independent substreams. Each substream is then transmitted simultaneously over each transmit antenna and, at the receiver, an optimal joint decoder is employed for retrieving the original symbol stream.

Since this strategy requires one single symbol stream, it can only be used for the transmission of a $\mathrm{SD}$ representation of the source.

\footnotetext{
${ }^{1}$ This is true also because the transmitted rate on each channel is the same, which is the only case of interest for us.
} 
The instantaneous capacity achievable with this strategy is given by (Foschini \& Gans, 1998)

$$
C=\log _{2} \operatorname{det}\left(I_{2}+\frac{\bar{\gamma}}{2} H H^{H}\right)
$$

where $I_{2}$ is the $2 \times 2$ identity matrix and $H^{H}$ denotes the conjugate transpose of the channel matrix $H$. In a similar way as before, given the outage probability the source coding rate $R_{S M}$ is chosen such that

$$
\operatorname{Pr}\left\{C<R_{S M}\right\}=P_{\text {out }}
$$

This CDF can be computed for a general MIMO channel without resorting to MonteCarlo simulation as indicated in (Chiani, Win \& Zanella, 2003). For the particular case of the $2 \times 2$ MIMO, a simple closed form expression is derived in the Appendix.

The expected distortion $D$ at the receiver is then

$$
D=\left(1-P_{\text {out }}\right) D_{1}+P_{\text {out }}
$$

where

$$
D_{1}=2^{-2 R_{S M}}
$$

is, as usual, the distortion achieved when the system is not in outage.

The outage rate $R_{S M}^{\text {out }}$ is given by

$$
R_{S M}^{\text {out }}=\left(1-P_{\text {out }}\right) R_{S M}
$$

\section{Zero-outage rate-adaptive source encoding (ZORA)}

In this section we assume that the source encoder knows the (instantaneous) value of the wireless channel mutual information. Thus, it encodes the source at a rate just below the mutual information, leading to the best achievable distortion at the receiver side. Note that this is a zero-outage strategy, that is expected to provide better results than the fixed-outage strategy, at the cost of increased system complexity due to the need for complete CSI at the transmitter side. Furthermore, this is a fast-adaptive technique compared to the fixed-outage, since the rate of adaptation is determined by the variations of the channel fading.

\subsection{Repetition}

Since transmitter side information does not increase capacity unless transmitted power is also adapted (Goldsmith, 2005), the capacity of this strategy in a given fading realization has the same expression as in (5) which can be rewritten using (1) as

$$
C=\frac{1}{2} \log _{2}\left(1+\frac{\bar{\gamma}}{2} \sum_{i, j=1}^{2} x_{i j}\right)=\frac{1}{2} \log _{2}\left(1+\frac{\bar{\gamma}}{2} x_{s}\right)
$$

where $\sum_{i, j=1}^{2} x_{i j}=x_{s} \sim \chi_{8}^{2}$ (Hogg \& Craig, 1970).

Since the transmitter has CSI knowledge, in every time instant the source coding rate $R_{R E P}$ can be adapted to achieve the instantaneous capacity $C$. The distortion $D_{r}$ observed at the receiver is then (Cover \& Thomas, 1991)

$$
D_{r}=2^{-2 R_{R E P}}=\frac{1}{1+\frac{\bar{\gamma}}{2} x_{S}}
$$


which is a continuous random variable. Its expected value is

$$
\begin{aligned}
D_{R E P}=\mathbb{E}\left[D_{r}\right] & =\int_{0}^{+\infty} \frac{1}{1+\frac{\bar{\gamma}}{2} z} f_{\chi}^{(8)}(z) d z \\
& =\frac{1}{48} \int_{0}^{+\infty} \frac{z^{3}}{2+\bar{\gamma} z} e^{-\frac{z}{2}} d z
\end{aligned}
$$

which yields

$$
D_{R E P}=\frac{1}{6} \cdot \frac{\bar{\gamma}-\bar{\gamma}^{2}+2 \bar{\gamma}^{3}-e^{\frac{1}{\bar{\gamma}}} \Gamma\left(0, \frac{1}{\bar{\gamma}}\right)}{\bar{\gamma}^{4}}
$$

The $\operatorname{CDF} F_{R E P}(d)$ of the distortion at the receiver can be derived as

$$
\begin{aligned}
F_{R E P}(d)=\operatorname{Pr}\left\{D_{r}<d\right\} & =\operatorname{Pr}\left\{x_{s}>\frac{2-2 d}{\bar{\gamma} d}\right\} \\
& =1-F_{\chi}^{(8)}\left(\frac{2-2 d}{\bar{\gamma} d}\right)
\end{aligned}
$$

\subsection{Alamouti}

This strategy (Zoffoli et al., 2008a) employs the Alamouti scheme to obtain two independent channels from the MIMO system. These two channels are then used for the transmission of a single description representation of the source, after demultiplexing it into two half-rate substreams. The capacity for the ALM strategy is given by (8) that, from (1), can be expressed as

$$
C=\log _{2}\left(1+\frac{\bar{\gamma}}{4} \sum_{i, j=1}^{2} x_{i j}\right)=\log _{2}\left(1+\frac{\bar{\gamma}}{4} x_{s}\right)
$$

where $\sum_{i, j=1}^{2} x_{i j}=x_{s} \sim \chi_{8}^{2}$ (Hogg \& Craig, 1970).

Using transmitter side information, the source coding rate $R_{A L M}$ can be adjusted to follow the variations of the capacity $C$. Thus, the distortion at the receiver is given by (Cover \& Thomas, 1991)

$$
D_{r}=2^{-2 R_{A L M}}=\frac{1}{\left(1+\frac{\bar{\gamma}}{4} x_{S}\right)^{2}}
$$

Its expected value can be evaluated as

$$
\begin{aligned}
D_{A L M}=\mathbb{E}\left[D_{r}\right] & =\int_{0}^{+\infty} \frac{1}{\left(1+\frac{\bar{\gamma}}{4} z\right)^{2}} f_{\chi}^{(8)}(z) d z \\
& =\frac{1}{6} \int_{0}^{+\infty} \frac{z^{3}}{(4+\bar{\gamma} z)^{2}} e^{-\frac{z}{2}} d z
\end{aligned}
$$

which finally results in

$$
D_{A L M}=\frac{2}{3} \cdot \frac{\bar{\gamma}[(\bar{\gamma}-4) \bar{\gamma}-4]+4 e^{\frac{2}{\bar{\gamma}}(3 \bar{\gamma}+2) \Gamma\left(0, \frac{2}{\bar{\gamma}}\right)}}{\bar{\gamma}^{5}}
$$


The CDF $F_{A L M}(d)$ of the distortion is

$$
\begin{aligned}
F_{A L M}(d)=\operatorname{Pr}\left\{D_{r}<d\right\} & =\operatorname{Pr}\left\{x_{s}>\frac{4-4 \sqrt{d}}{\bar{\gamma} \sqrt{d}}\right\} \\
& =1-F_{\chi}^{(8)}\left(\frac{4-4 \sqrt{d}}{\bar{\gamma} \sqrt{d}}\right)
\end{aligned}
$$

\subsection{Spatial multiplexing strategy}

Here, a single description of the source, i.e. a single symbol stream, is first demultiplexed and encoded into two separate and independent substreams. Each substream is then transmitted simultaneously over each transmit antenna and, at the receiver, an optimal joint decoder is employed for retrieving the original symbol stream. The capacity of this strategy is given by (10).

In the Appendix it is shown that the expected distortion for ZORA over SM MIMO system is given by

$$
\begin{aligned}
& D_{S M}=-\frac{16\left[\bar{\gamma}-(\bar{\gamma}+2) e^{\frac{2}{\bar{\gamma}}} \Gamma\left(0, \frac{2}{\bar{\gamma}}\right)\right]^{2}}{\bar{\gamma}^{6}} \\
&+\frac{8\left[\bar{\gamma}-2 e^{\frac{2}{\bar{\gamma}}} \Gamma\left(0, \frac{2}{\bar{\gamma}}\right)\right]\left[\bar{\gamma}(\bar{\gamma}+2)-4(\bar{\gamma}+1) e^{\frac{2}{\bar{\gamma}}} \Gamma\left(0, \frac{2}{\bar{\gamma}}\right)\right]}{\bar{\gamma}^{6}} .
\end{aligned}
$$

The CDF of the distortion observed at the receiver is obtained in the Appendix as

$$
\begin{aligned}
F_{S M}(d)=\operatorname{Pr}\left\{D_{r}<d\right\} & =1-\operatorname{Pr}\left\{x_{1} x_{2}<\frac{1}{\sqrt{d}}\right\} \\
& =1-F\left(\frac{1}{\sqrt{d}}\right)
\end{aligned}
$$

where

$$
\begin{aligned}
F(z)=\frac{2}{\bar{\gamma}^{3}} e^{\frac{4}{\bar{\gamma}}} \int_{1}^{z} e^{-\frac{2}{\bar{\gamma}} x_{2}} \cdot\left\{e^{-\frac{2}{\bar{\gamma}}}\left[\bar{\gamma}^{2}-2 \bar{\gamma}\left(x_{2}-1\right)+2\left(x_{2}-1\right)^{2}\right]\right. \\
\left.-e^{-\frac{2}{\bar{\gamma}} \frac{z}{x_{2}}}\left[\bar{\gamma}^{2}+2 \bar{\gamma}\left(\frac{z}{x_{2}}-x_{2}\right)+2\left(\frac{z}{x_{2}}-x_{2}\right)^{2}\right]\right\} d x_{2}
\end{aligned}
$$

\section{Numerical results}

\subsection{Discussion for the fixed-outage strategies}

We begin the discussion by comparing only the three TS-MD strategies. Then, we compare TS-MD-OPT with the remaining three strategies.

Figure 2 compares the expected distortions achievable with the TS strategies at a fixed $\bar{\gamma}$ of $10 \mathrm{~dB}$. These results can be explained using the same observations we made in (Zoffoli et al., 2008b), where we considered MD strategies over two parallel and independent fading channels. For completeness, we now briefly restate here these conclusions.

As expected, TS-MD-OPT achieves the lowest distortions, since it is designed to minimize Eq. (7). At low outage probabilities, both descriptions are correctly decoded most of the time 


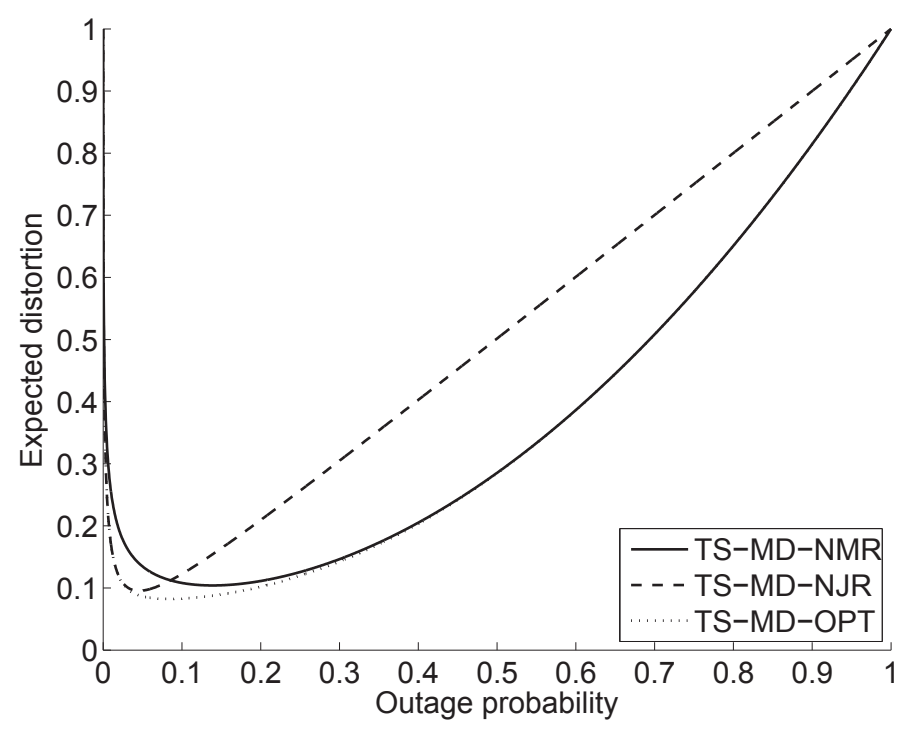

Fig. 2. FORA: expected distortion vs. outage probability for the different TS strategies with $\bar{\gamma}$ $=10 \mathrm{~dB}$.

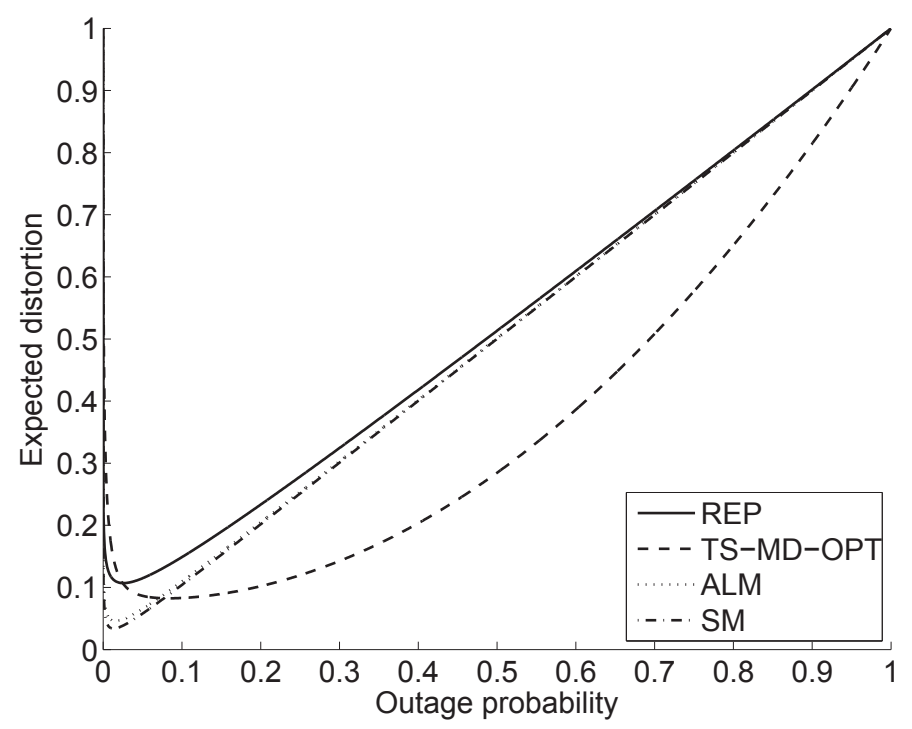

Fig. 3. FORA: expected distortion vs. outage probability for the different strategies with $\bar{\gamma}=$ $10 \mathrm{~dB}$. 
and optimal performance is achievable with the TS-MD-NJR strategy, since it is designed to minimize the distortion $D_{0}$. As the outage probability gets higher, the receiver becomes able to correctly decode only one description most of the time and TS-MD-NMR achieves optimal performance, since it is designed to minimize the distortion $D_{1}$.

Figure 3 compares the remaining strategies and TS-MD-OPT at a fixed $\bar{\gamma}$ of $10 \mathrm{~dB}$. As can be seen, the lowest distortions are achieved with the SM strategy. However, this performance comes at the expense of complexity, mainly due to the presence of the joint decoder at the receiver. Interestingly, the ALM strategy, which can be employed for reducing this complexity, shows only a very small loss in performance with respect to SM. Looking at the source coding rates of the different strategies, reported in Fig. 4, it can be seen that ALM obtains rather high coding rates, but still significantly lower than those of SM.

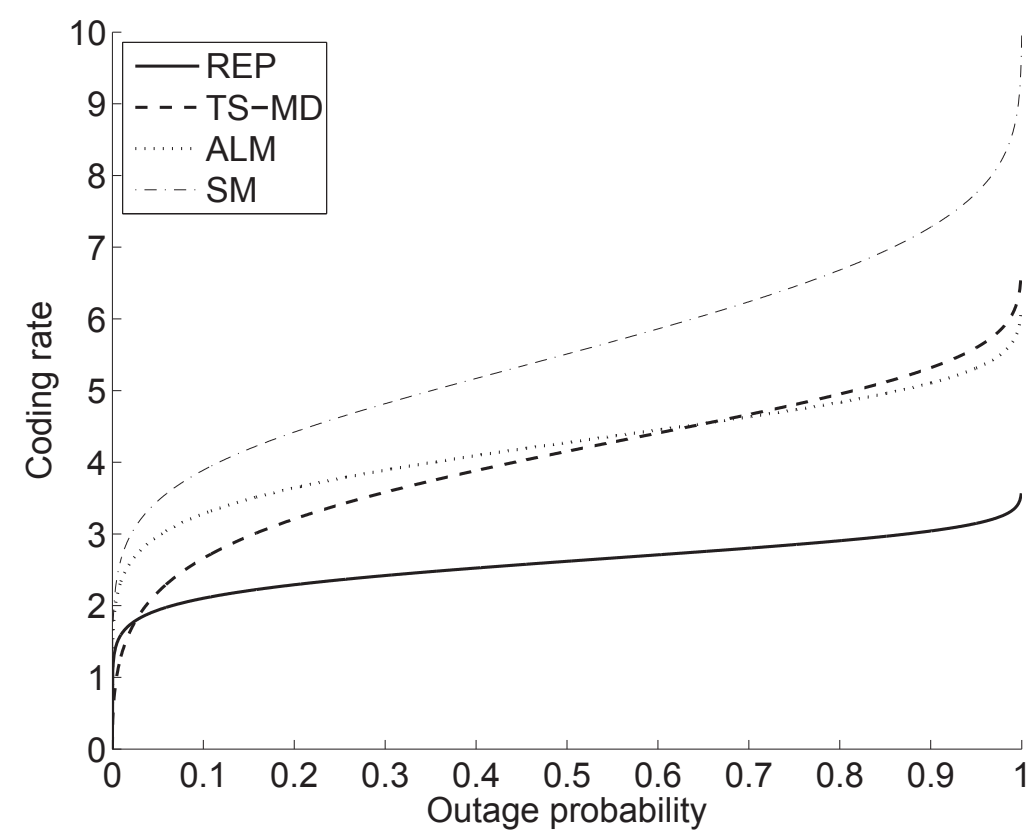

Fig. 4. FORA: source coding rates vs. outage probability for the different strategies with $\bar{\gamma}=$ $10 \mathrm{~dB}$.

This observation, at a first analysis, might erroneously lead to the expectation of a more evident difference in performance between these two strategies. In fact, it must be recalled that the distortions $D_{1}$ are exponential decaying functions of the coding rate (see Eqs. (9) and (11)). So, due to this type of dependency, the distortions $D_{1}$ are very similar even though the coding rates are significantly different.

Returning to Fig. 3, as the outage probability grows, performance of SM and ALM quickly worsen and the lowest distortions become achievable with the TS-MD-OPT strategy. This happens because SM and ALM are both transmitting over a single unreliable channel, while TS-MD-OPT employs path diversity over two independent and equally unreliable channels reducing the overall system outage probability. The REP strategy has in general the worst 
performance, except for very low values of outage probabilities where it performs slightly better than TS-MD-OPT.

We now consider the outage rates achievable with the various strategies, plotted in Fig. 5. In

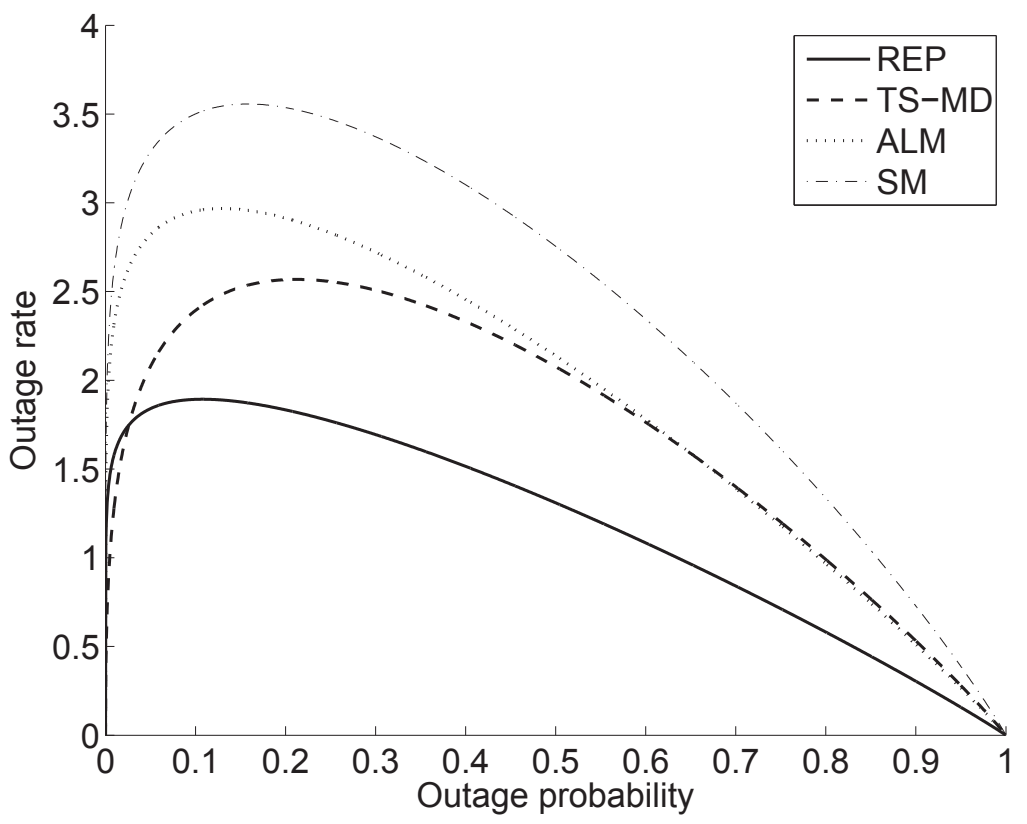

Fig. 5. FORA: outage rate vs. outage probability for the different strategies with $\bar{\gamma}=10 \mathrm{~dB}$.

(Choudhury \& Gibson, 2007) it has been shown that, when considering the lossy transmission of information over a single channel with very slow Rayleigh fading, designing the system to maximize outage rate does not lead to the minimization of the distortion at the receiver. Inspired by this observation, we want to determine if the same result applies to our MIMO case.

We denote by $p_{d}$ the outage probability that minimizes expected distortion and by $p_{r}$ the outage probability that maximizes outage rate. Table 1 shows the values of $p_{d}, p_{r}$, the corresponding distortions and the percent differences in distortion for the various strategies with $\bar{\gamma}=10 \mathrm{~dB}$, obtained from Figs. 3 and 5. As can be seen, the outage probabilities that minimize distortion are very different from the outage probabilities that maximize outage

\begin{tabular}{|c|c|c|c|c|c|}
\hline & $p_{\boldsymbol{d}}$ & $\boldsymbol{p}_{\boldsymbol{r}}$ & $\boldsymbol{D}\left(\boldsymbol{p}_{\boldsymbol{d}}\right)$ & $\boldsymbol{D}\left(\boldsymbol{p}_{\boldsymbol{r}}\right)$ & $\boldsymbol{\Delta} \boldsymbol{D} \%$ \\
\hline ALM & 0.015 & 0.130 & 0.0458 & 0.1372 & 199.41 \\
\hline TS-MD-OPT & 0.084 & 0.212 & 0.0825 & 0.1058 & 28.24 \\
\hline REP & 0.026 & 0.108 & 0.1069 & 0.1551 & 45.01 \\
\hline SM & 0.013 & 0.157 & 0.0338 & 0.1589 & 369.76 \\
\hline
\end{tabular}

Table 1. $p_{d}, p_{r}$ and respective distortions for the various strategies with $\bar{\gamma}=10 \mathrm{~dB}$. 
rate. Thus, if the system is designed to maximize outage rate instead of minimizing distortion, suboptimal performance is achieved. In some cases this approach could result in much higher distortions, as in the SM strategy where the percent difference between the two distortions is almost $370 \%$. TS-MD-OPT is the less sensitive strategy to this design error, but still has a distortion that is more than $28 \%$ higher than the minimum distortion.

\subsection{Discussion for the zero-outage strategies}

Figure 6 plots the expected distortion as a function of $\bar{\gamma}$ for the different strategies. As can be seen, the lowest distortion is achieved with the SM strategy at all values of $\bar{\gamma}$. This performance, however, comes at the expense of realization complexity, due to the presence of the joint ML decoder at the receiver (Zoffoli et al., 2008a). Similarly to the case of CSI at the receiver only of (Zoffoli et al., 2008a), if the ALM strategy is employed to reduce this complexity, only a small decrease in performance is observed, especially at high values of $\bar{\gamma}$. Significantly higher distortion is produced with the REP strategy.

Figures 7, 8 and 9 plot the CDF of the distortion for different values of $\bar{\gamma}$ respectively for the REP, ALM and SM strategies. The square markers in the plots represent the value of the expected distortion for the respective value of $\bar{\gamma}$. Interestingly, an increase in the value of $\bar{\gamma}$ not only improves the value of expected distortion for every strategy (as it appears evident also from Fig. 6), but also improves the probability $p_{e}(\bar{\gamma})$ of achieving that distortion. The values of $p_{e}(\bar{\gamma})$ for the different strategies and for different values of $\bar{\gamma}$ are reported in Table 2. It can

\begin{tabular}{|c|c|c|c|c|}
\hline & \multicolumn{4}{|c|}{$p_{e}(\bar{\gamma})$} \\
\hline & $\bar{\gamma}=\mathbf{1} \mathbf{d B}$ & $\bar{\gamma}=\mathbf{3} \mathbf{d B}$ & $\bar{\gamma}=\mathbf{5} \mathbf{d B}$ & $\bar{\gamma}=\mathbf{1 0} \mathbf{~ d B}$ \\
\hline ALM & 0.630 & 0.658 & 0.684 & 0.731 \\
\hline REP & 0.594 & 0.608 & 0.621 & 0.637 \\
\hline SM & 0.641 & 0.678 & 0.712 & 0.787 \\
\hline
\end{tabular}

Table 2. Probability of achieving expected distortion for the various strategies with different values of $\bar{\gamma}$.

be observed that, given the same increase of $\bar{\gamma}$, the increase in $p_{e}(\bar{\gamma})$ in the REP strategy is significantly lower than the increase in $p_{e}(\bar{\gamma})$ for the remaining two strategies. For example, if $\bar{\gamma}$ increases from $1 \mathrm{~dB}$ to $10 \mathrm{~dB}, p_{e}(\bar{\gamma})$ in the REP strategy increases by about 0.04 , while in ALM and SM it increases by about 0.10 and 0.14 , respectively. Moreover, returning to Figs. 7 , 8 and 9, an increase of $\bar{\gamma}$ also causes an increase in the slope of the CDF for all strategies, suggesting that the values of distortion become less variable as $\bar{\gamma}$ increases.

A comparison of the CDF of the distortion for the various strategies at a fixed $\bar{\gamma}$ of $5 \mathrm{~dB}$ is reported in Fig. 10. Both ALM and SM strategies have similar and very steep CDFs, which means that it is possible with these strategies to achieve low values of distortion with high probability. For example, with a probability of $0.9, \mathrm{SM}$ achieves a distortion approximately equal to 0.05 , while ALM achieves a distortion approximately equal to 0.07 . The REP strategy has a much less steep CDF than the other two strategies, and indeed, with a probability of 0.9 it achieves a significantly higher distortion, approximately equal to 0.15 .

\subsection{Comparison between fixed-outage and zero-outage}

The zero outage strategies require CSI at the transmitter, fast adaptation at the transmitter to respond to the CSI, and commensurate additional complexity compared to the fixed outage 


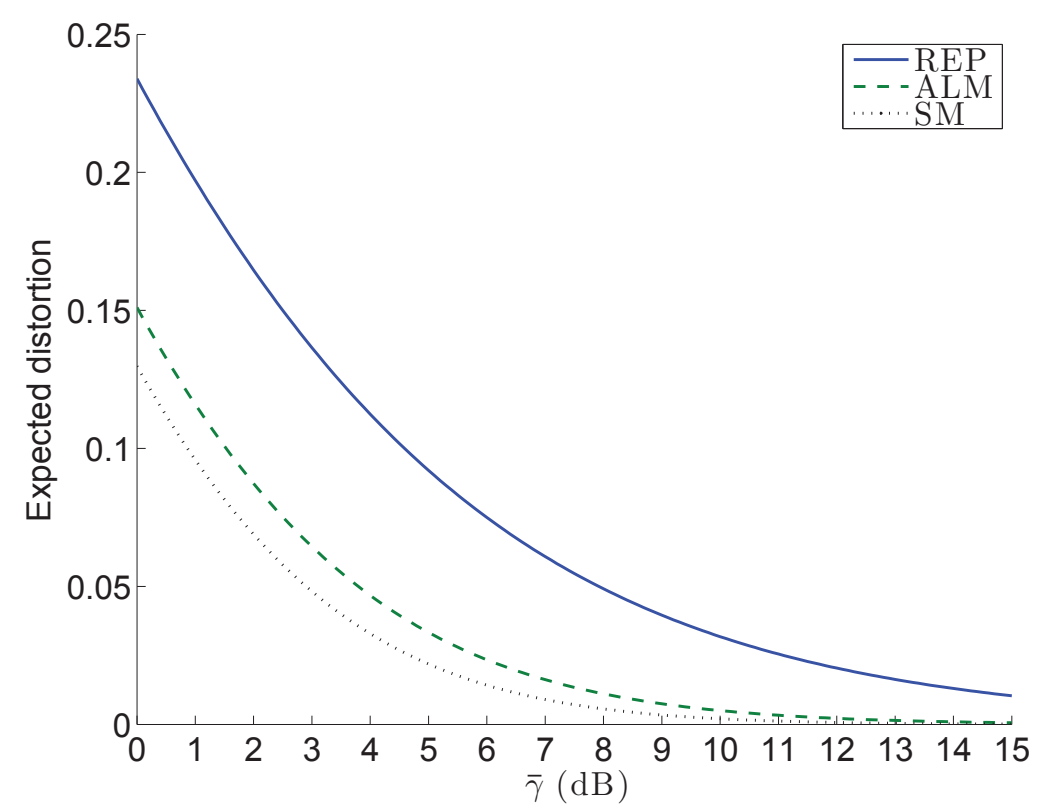

Fig. 6. ZORA: expected distortion as a function of $\bar{\gamma}$ for the different strategies.

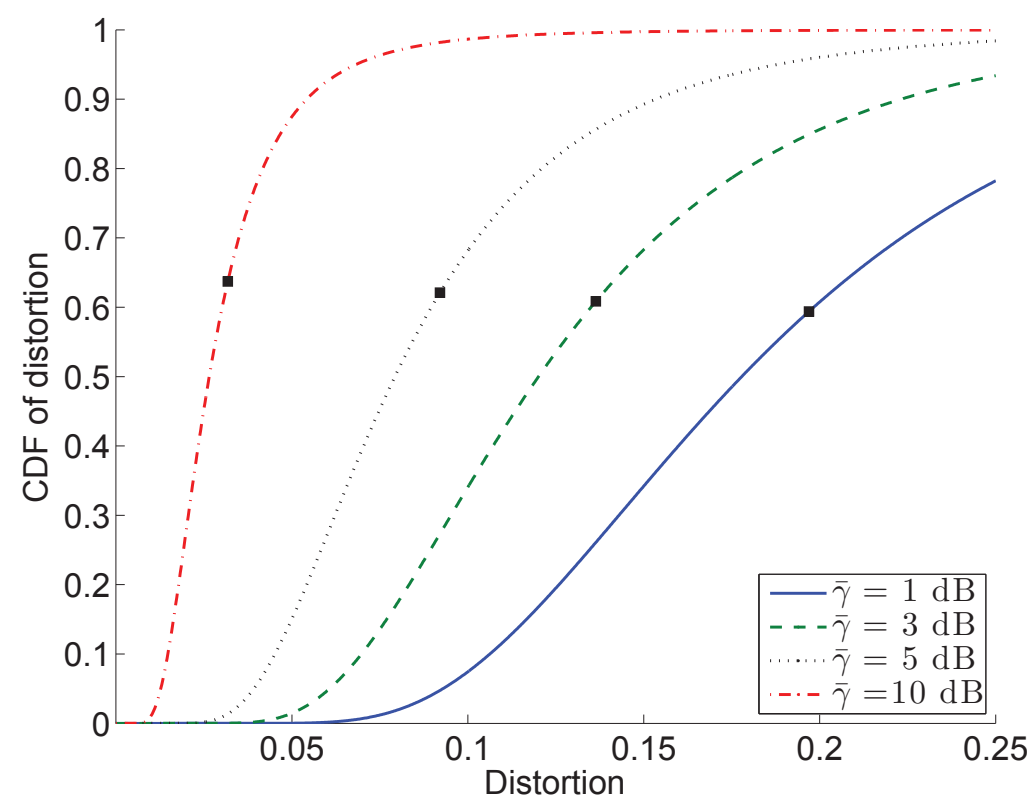

Fig. 7. ZORA: CDF of distortion for REP strategy with different values of $\bar{\gamma}$. 


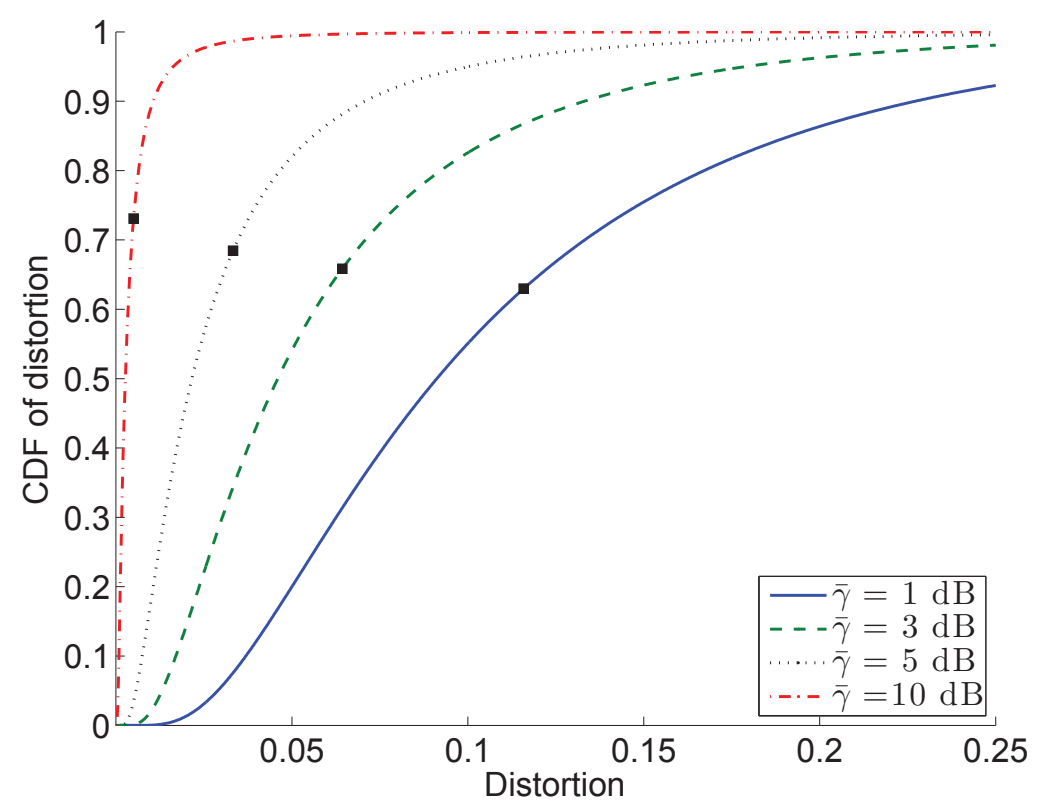

Fig. 8. ZORA: CDF of distortion for ALM strategy with different values of $\bar{\gamma}$.

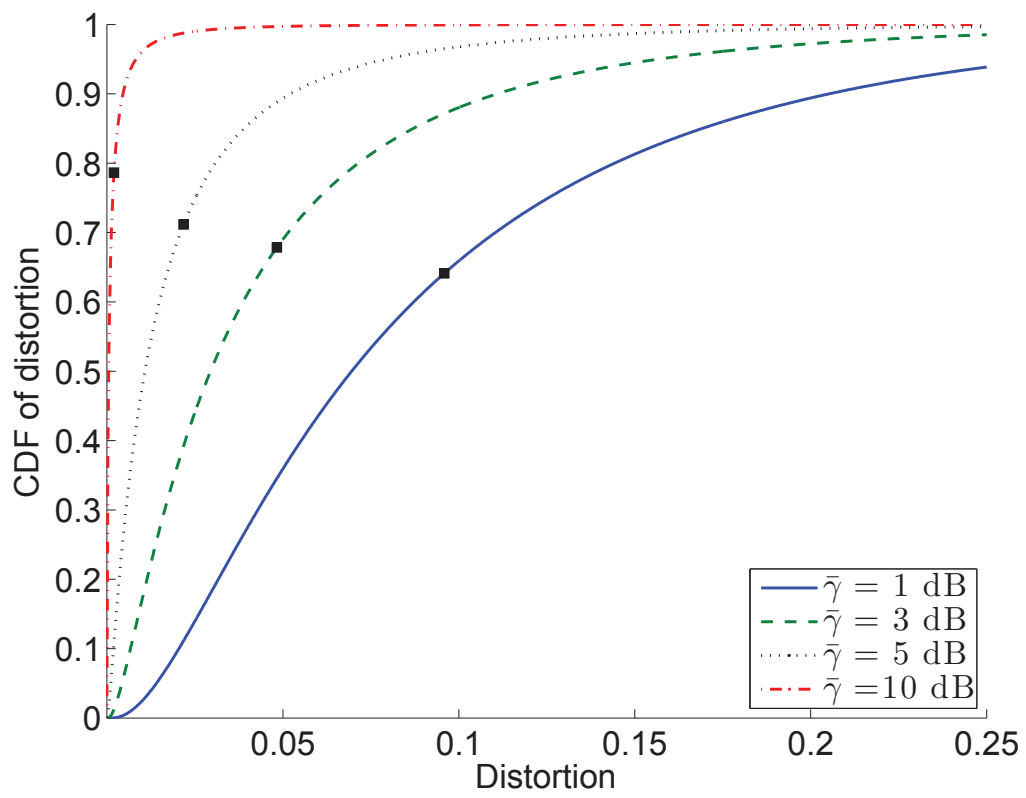

Fig. 9. ZORA: CDF of distortion for SM strategy with different values of $\bar{\gamma}$. 


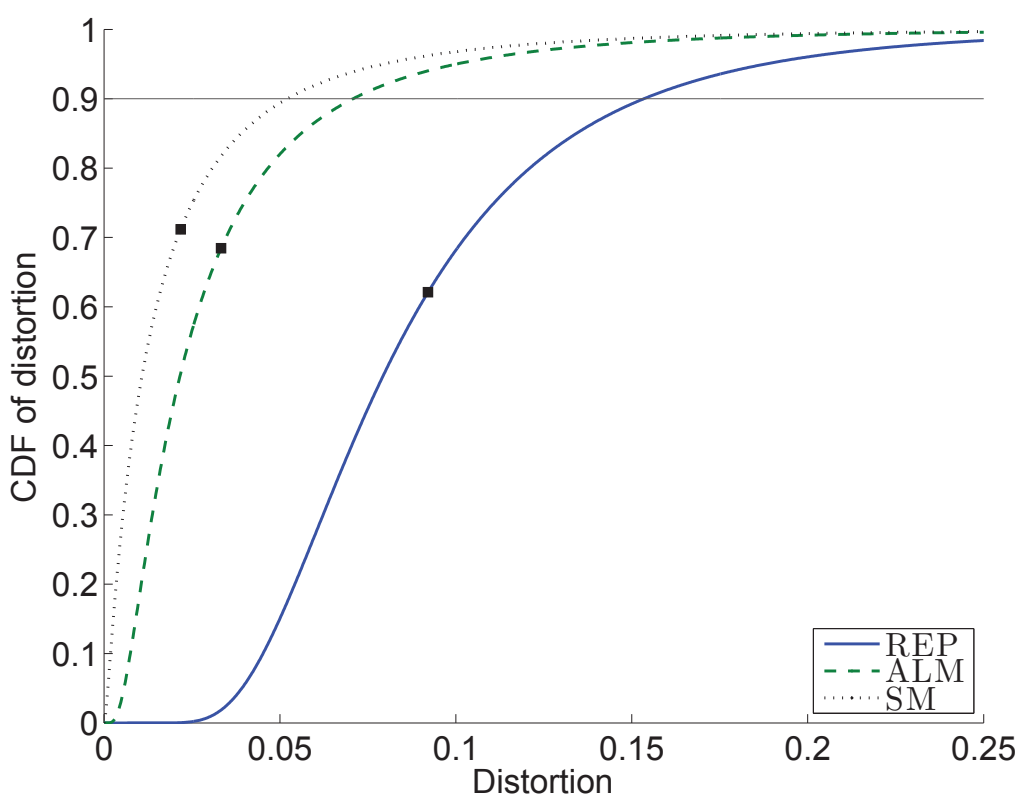

Fig. 10. ZORA: CDF of the distortion at the receiver for the different strategies at a fixed $\bar{\gamma}$ of $5 \mathrm{~dB}$.

approaches. As a result, we would hope for and expect some gain in performance. Using SNR to denote the ratio $P_{t} / N$, a comparison of the FORA minimum distortion values in Fig. 3 for an SNR of $10 \mathrm{~dB}$, with the minimum expected distortion achieved by the Alamouti and Spatial Multiplexing schemes at a $10 \mathrm{~dB}$ SNR, available in Fig. 6 for ZORA, reveals that the more complex zero outage rate strategies have more than an order of magnitude lower distortion. In fact, the Alamouti scheme under the zero outage approach achieves the same minimum average distortion as in Fig. 3 but at less than a $6 \mathrm{~dB}$ SNR, and the spatial multiplexing scheme under ZORA produces the minimum average distortion in Fig. 3 but at an SNR of around 4 $\mathrm{dB}$. Thus, 4 to $6 \mathrm{~dB}$ gains in SNR are available with the more complex zero outage strategies compared to the fixed outage methods.

\section{Conclusions}

The results for the Fixed Outage Rate strategies used in a TS MIMO mode show that of the three MD methods, namely, the No Excess Joint Rate coder (MD-NJR), the No Excess Marginal Rate coder (MD-NMR), and the optimized MD coder (MD-OPT), the MD-OPT performs best across all outage probabilities since if follows the envelope of the other two methods. However, to optimize the MD-OPT coder, we need to know the packet loss rate to minimize the average distortion. While the TS-MD-OPT outperforms the other MD approaches studied, the TS-MD-OPT scheme performs worse for low outage probabilities than the REP, ALM, and SM MIMO schemes that use an SD coder and is only able to take advantage of the MD source coding method as the outage probability moves toward 0.1 and higher. Perhaps the most important result obtained is that the outage probability that maximizes outage rate 
is much different from the outage probability that minimizes expected distortion for all of the methods, and that the performance penalty for ignoring the expected distortion in the optimization can be substantial. Thus, the usual approach of selecting the outage probability to maximize outage rate is suboptimal for lossy source coding.

For the ZORA methods, wherein CSI is available at the transmitter and the source coding rate is adapted to match the instantaneous channel capacity, it is shown that SM performs best among REP, ALM, and SM, but that ALM performs very well, particularly in light of its lower complexity. Plots of the cumulative distribution of the distortion as a function of SNR indicate that not only does an increase in SNR reduce the expected distortion, but it also increases the probability that that distortion will be achieved. Additionally, the CDFs of the distortion have a much steeper slope with increased SNR, thus implying a much narrower range of variation for the performance. The more complex and adaptive ZORA approach using transmitter CSI is also shown to achieve more than an order of magnitude reduction in average distortion over the FORA methods and to yield a reduction of 4 to $6 \mathrm{~dB}$ in required SNR for the same performance.

\section{Appendix: Statistics of the distortion for ZORA}

To determine the expression of the expected distortion at the receiver for the zero-outage strategy, we first need to introduce the characteristic function $\phi_{C}(z)$ of the capacity $C$, defined as (Chiani, Win \& Zanella, 2003)

$$
\phi_{C}(z)=\mathbb{E}\left[e^{j 2 \pi C z}\right]=K \operatorname{det}[U(z)]
$$

where, for a $2 \times 2$ uncorrelated MIMO Rayleigh fading channel, $K=1$ and $U(z)$ is a $2 \times 2$ matrix with $i k$-th elements given by (Chiani, Win \& Zanella, 2003)

$$
u_{i k}(z)=\int_{0}^{+\infty} x^{i+k-2} e^{-x}\left(1+\frac{\bar{\gamma}}{2} x\right)^{j \frac{2 \pi z}{\ln 2}} d x
$$

Since the source coding rate $R_{S M}$ is adapted, in every time instant, to the capacity $C$, we can now express the expected distortion $D_{S M}$ in terms of the function $\phi_{C}(z)$ as (Cover \& Thomas, 1991)

$$
\begin{aligned}
D_{S M} & =\mathbb{E}\left[2^{-2 R_{S M}}\right]=\mathbb{E}\left[e^{-2 C \ln 2}\right]=\phi_{C}\left(j \frac{\ln 2}{\pi}\right) \\
& =\operatorname{det}\left[U\left(j \frac{\ln 2}{\pi}\right)\right]=\hat{u}_{11} \hat{u}_{22}-\hat{u}_{12} \hat{u}_{21}
\end{aligned}
$$

where we defined $\hat{u}_{i k}=u_{i k}(j \ln 2 / \pi)$. Developing the expression in (13), we get for the $2 \times 2$ MIMO 


$$
\begin{aligned}
& \hat{u}_{11}=2 \cdot \frac{\bar{\gamma}-2 e^{\frac{2}{\bar{\gamma}}} \Gamma\left(0, \frac{2}{\bar{\gamma}}\right)}{\bar{\gamma}^{2}} \\
& \hat{u}_{12}=4 \cdot \frac{(2+\bar{\gamma}) e^{\frac{2}{\bar{\gamma}}} \Gamma\left(0, \frac{2}{\bar{\gamma}}\right)-\bar{\gamma}}{\bar{\gamma}^{3}} \\
& \hat{u}_{21}=\hat{u}_{12} \\
& \hat{u}_{22}=4 \cdot \frac{\bar{\gamma}(2+\bar{\gamma})-4(1+\bar{\gamma}) e^{\frac{2}{\bar{\gamma}}} \Gamma\left(0, \frac{2}{\bar{\gamma}}\right)}{\bar{\gamma}^{4}}
\end{aligned}
$$

and, after substituting these expressions into Eq. (14), we derive the final expression of $D_{S M}$ as

$$
\begin{aligned}
& D_{S M}=-\frac{16\left[\bar{\gamma}-(\bar{\gamma}+2) e^{\frac{2}{\bar{\gamma}}} \Gamma\left(0, \frac{2}{\bar{\gamma}}\right)\right]^{2}}{\bar{\gamma}^{6}} \\
&+\frac{8\left[\bar{\gamma}-2 e^{\frac{2}{\bar{\gamma}}} \Gamma\left(0, \frac{2}{\bar{\gamma}}\right)\right]\left[\bar{\gamma}(\bar{\gamma}+2)-4(\bar{\gamma}+1) e^{\frac{2}{\bar{\gamma}}} \Gamma\left(0, \frac{2}{\bar{\gamma}}\right)\right]}{\bar{\gamma}^{6}}
\end{aligned}
$$

The CDF $F_{S M}(d)$ of the distortion at the receiver is related to that of the capacity via the relationship

$$
F_{S M}(d)=\operatorname{Pr}\left\{D_{r}<d\right\}=1-\operatorname{Pr}\left\{C>\log _{2} \frac{1}{\sqrt{d}}\right\} .
$$

For MIMO, the CDF of the capacity can be derived starting from the characteristic function (Chiani, Win \& Zanella, 2003; Shin et al., 2006). For small MIMO systems results for the PDF and CDF can also be found in (Smith et al., 2003).

For the particular case of the $2 \times 2$ MIMO of interest here, we derive below a closed form expression for the CDF. We first need to rewrite the expression of the capacity in (10) using the singular value decomposition of $H$, to obtain (Telatar, 1999; Winters, 1987)

$$
C=\log _{2} \prod_{i=1}^{2}\left(1+\frac{\bar{\gamma}}{2} \lambda_{i}\right)=\log _{2}\left(x_{1} x_{2}\right)
$$

where

$$
x_{i}=1+\frac{\bar{\gamma}}{2} \lambda_{i}, \quad i=1,2
$$

and the $\lambda_{1} \geq \lambda_{2} \geq 0$ are the two ordered nonzero eigenvalues of the matrix $H H^{H}$, giving $x_{i}>1$ and $x_{1} \geqslant x_{2}$.

The distortion observed at the receiver is then (Cover \& Thomas, 1991)

$$
D_{r}=2^{-2 R_{S M}}=\frac{1}{\left(x_{1} x_{2}\right)^{2}}
$$


and $F_{S M}(d)$ results

$$
\begin{aligned}
F_{S M}(d)=\operatorname{Pr}\left\{D_{r}<d\right\} & =1-\operatorname{Pr}\left\{x_{1} x_{2}<\frac{1}{\sqrt{d}}\right\} \\
& =1-F\left(\frac{1}{\sqrt{d}}\right)
\end{aligned}
$$

where $F(z)=\operatorname{Pr}\left\{x_{1} x_{2}<z\right\}$.

We now define $S$ as the set of points $\left(x_{1}, x_{2}\right)$ such that

$$
S=\left\{\left(x_{1}, x_{2}\right) \in \mathbb{R}^{2}: x_{1}>1 ; x_{2}>1 ; x_{1} \geqslant x_{2} ; x_{1} x_{2}<z\right\}
$$

If $X$ is the vector $X=\left(x_{1}, x_{2}\right)$ with joint PDF $f_{X}\left(x_{1}, x_{2}\right), F(z)$ can then be evaluated as

$$
\begin{aligned}
F(z) & =\int_{S} f_{X}\left(x_{1}, x_{2}\right) d x_{1} d x_{2} \\
& =\frac{1}{2} \int_{1}^{z} \int_{1}^{\frac{z}{x_{2}}} f_{X}\left(x_{1}, x_{2}\right) d x_{1} d x_{2}
\end{aligned}
$$

The joint PDF of $X$ can be expressed in terms of the joint PDF $f_{\Lambda}\left(\lambda_{1}, \lambda_{2}\right)$ of the vector of the eigenvalues $\Lambda=\left(\lambda_{1}, \lambda_{2}\right)$ as (Papoulis \& Pillai, 2001)

$$
f_{X}\left(x_{1}, x_{2}\right)=\frac{f_{\Lambda}\left(\lambda_{1}, \lambda_{2}\right)}{\operatorname{det}(J)}=\frac{4}{\bar{\gamma}^{2}} f_{\Lambda}\left(\lambda_{1}, \lambda_{2}\right)
$$

where $J$ is the Jacobian matrix of $X$. Since from Eq. (15) we have

$$
\lambda_{i}=\frac{2}{\bar{\gamma}}\left(x_{i}-1\right), \quad i=1,2
$$

we can rewrite Eq. (18) as

$$
f_{X}\left(x_{1}, x_{2}\right)=\frac{4}{\bar{\gamma}^{2}} f_{\Lambda}\left(\frac{2}{\bar{\gamma}}\left(x_{1}-1\right), \frac{2}{\bar{\gamma}}\left(x_{2}-1\right)\right)
$$

The joint PDF of the eigenvalues of $\mathrm{HH}^{H}$ for a MIMO system with uncorrelated fading between antenna elements can be written as (Chiani, Win \& Zanella, 2003; Chiani, Win, Zanella, Mallik \& Winters, 2003; Telatar, 1999)

$$
f_{\Lambda}\left(\lambda_{1}, \lambda_{2}\right)=e^{-\lambda_{1}} e^{-\lambda_{2}}\left(\lambda_{1}-\lambda_{2}\right)^{2}
$$

which, substituting into Eq. (19), yields

$$
f_{X}\left(x_{1}, x_{2}\right)=\frac{16}{\bar{\gamma}^{4}} e^{\frac{4}{\bar{\gamma}}} e^{-\frac{2}{\bar{\gamma}}\left(x_{1}+x_{2}\right)}\left(x_{1}-x_{2}\right)^{2}
$$

Substituting this last expression into (17) gives

$$
F(z)=\frac{8}{\bar{\gamma}^{4}} e^{\frac{4}{\bar{\gamma}}} \int_{1}^{z} e^{-\frac{2}{\bar{\gamma}} x_{2}} \int_{1}^{\frac{z}{x_{2}}} e^{-\frac{2}{\bar{\gamma}} x_{1}}\left(x_{1}-x_{2}\right)^{2} d x_{1} d x_{2}
$$


which can be finally written as

$$
\begin{aligned}
& F(z)=\frac{2}{\bar{\gamma}^{3}} e^{\frac{4}{\bar{\gamma}}} \int_{1}^{z} e^{-\frac{2}{\bar{\gamma}} x_{2}} \\
& \cdot\left\{e^{-\frac{2}{\bar{\gamma}}\left[\bar{\gamma}^{2}-2 \bar{\gamma}\left(x_{2}-1\right)+2\left(x_{2}-1\right)^{2}\right]}\right. \\
&\left.\quad-e^{-\frac{2}{\bar{\gamma}} \frac{z}{x_{2}}}\left[\bar{\gamma}^{2}+2 \bar{\gamma}\left(\frac{z}{x_{2}}-x_{2}\right)+2\left(\frac{z}{x_{2}}-x_{2}\right)^{2}\right]\right\} d x_{2}
\end{aligned}
$$

By using this expression into (16), it is then possible to evaluate the CDF of distortion for the SM strategy.

\section{References}

Alamouti, S. (1998). A simple transmit diversity technique for wireless communications, IEEE J. Sel. Areas Commun. 16(8): 1451-1458.

Balam, J. \& Gibson, J. (2006). Path diversity and multiple descriptions with rate dependent packet losses, Proc. Information Theory and Applications Workshop, University of California, San Diego, La Jolla, CA.

Chiani, M., Win, M. Z. \& Zanella, A. (2003). On the capacity of spatially correlated MIMO Rayleigh fading channels, IEEE Trans. on Information Theory, 49(10): 2363-2371.

Chiani, M., Win, M. Z., Zanella, A., Mallik, R. K. \& Winters, J. H. (2003). Bounds and approximations for optimum combining of signals in the presence of multiple co-channel interferers and thermal noise, IEEE Trans. on Communications 51(2): 296-307.

Choudhury, S. \& Gibson, J. D. (2007). Information transmission over fading channels, Global Telecommunications Conference, 2007. GLOBECOM '07. IEEE pp. 3316-3321.

Cover, T. \& Thomas, J. (1991). Elements of Information Theory, John Wiley \& Sons.

Effros, M., Koetter, R., Goldsmith, A. \& Medard, M. (2004). On source and channel codes for multiple inputs and outputs: does multiple description beat space time?, Information Theory Workshop, 2004. IEEE pp. 324-329.

Foschini, G. J. (1996). Layered space-time architecture for wireless communication a fading environment when using multiple antennas, Bell Labs Tech. J. 1(2): 41-59.

Foschini, G. J. \& Gans, M. J. (1998). On limits of wireless communications in a fading environment when using multiple antennas, Wirel. Pers. Commun. 6(3): 311-335.

Gamal, A. \& Cover, T. (1982). Achievable rates for multiple descriptions, Information Theory, IEEE Transactions on 28(6): 851-857.

Goldsmith, A. (2005). Wireless communications, Cambridge University Press.

Hogg, R. V. \& Craig, A. T. (1970). Introduction to Mathematical Statistics, 3rd edn, NY: Macmillan.

Papoulis, A. \& Pillai, S. (2001). Probability, Random Variables and Stochastic Processes, 4th edn, McGraw-Hill Science.

Sandhu, S. \& Paulraj, A. (2000). Space-time block codes: a capacity perspective, IEEE Commun. Lett. 4(12): 384-386.

Shin, H., Win, M., Lee, J. H. \& Chiani, M. (2006). On the capacity of doubly correlated MIMO channels, IEEE Trans. on Wireless Commun. 5(8): 2253-2266.

Smith, P., Garth, L. \& Loyka, S. (2003). Exact capacity distributions for MIMO systems with small numbers of antennas, IEEE Communications Letters 7(10): 481-483. 
Telatar, E. (1999). Capacity of multi-antenna Gaussian channels, Europ. Trans. on Telecomm. 10(6): 585-595.

Tse, D. \& Viswanath, P. (2006). Fundamentals of Wireless Communication, Cambridge University Press.

Winters, J. H. (1987). On the capacity of radio communication systems with diversity in Rayleigh fading environment, IEEE J. Sel. Areas Commun. SAC-5(5): 871-878.

Zoffoli, M., Gibson, J. D. \& Chiani, M. (2008a). On strategies for source information transmission over MIMO systems, Global Telecommunications Conference, 2008. GLOBECOM '08. IEEE.

Zoffoli, M., Gibson, J. D. \& Chiani, M. (2008b). Source coding diversity and multiplexing strategies for a 2x2 MIMO system, Information Theory and Applications Workshop, University of California, San Diego, La Jolla, CA. 


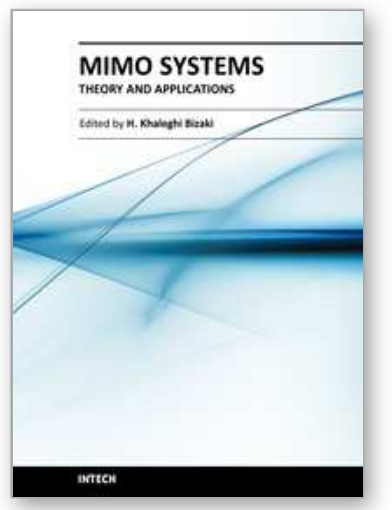

\author{
MIMO Systems, Theory and Applications \\ Edited by Dr. Hossein Khaleghi Bizaki
}

ISBN 978-953-307-245-6

Hard cover, 488 pages

Publisher InTech

Published online 04, April, 2011

Published in print edition April, 2011

In recent years, it was realized that the MIMO communication systems seems to be inevitable in accelerated evolution of high data rates applications due to their potential to dramatically increase the spectral efficiency and simultaneously sending individual information to the corresponding users in wireless systems. This book, intends to provide highlights of the current research topics in the field of MIMO system, to offer a snapshot of the recent advances and major issues faced today by the researchers in the MIMO related areas. The book is written by specialists working in universities and research centers all over the world to cover the fundamental principles and main advanced topics on high data rates wireless communications systems over MIMO channels. Moreover, the book has the advantage of providing a collection of applications that are completely independent and self-contained; thus, the interested reader can choose any chapter and skip to another without losing continuity.

\title{
How to reference
}

In order to correctly reference this scholarly work, feel free to copy and paste the following:

Marco Zoffoli, Jerry D. Gibson and Marco Chiani (2011). Rate-Adaptive Information Transmission over MIMO Channels, MIMO Systems, Theory and Applications, Dr. Hossein Khaleghi Bizaki (Ed.), ISBN: 978-953-307245-6, InTech, Available from: http://www.intechopen.com/books/mimo-systems-theory-and-applications/rateadaptive-information-transmission-over-mimo-channels

\section{INTECH}

open science | open minds

\section{InTech Europe}

University Campus STeP Ri

Slavka Krautzeka 83/A

51000 Rijeka, Croatia

Phone: +385 (51) 770447

Fax: +385 (51) 686166

www.intechopen.com

\section{InTech China}

Unit 405, Office Block, Hotel Equatorial Shanghai

No.65, Yan An Road (West), Shanghai, 200040, China

中国上海市延安西路65号上海国际贵都大饭店办公楼 405 单元

Phone: +86-21-62489820

Fax: $+86-21-62489821$ 
(C) 2011 The Author(s). Licensee IntechOpen. This chapter is distributed under the terms of the Creative Commons Attribution-NonCommercialShareAlike-3.0 License, which permits use, distribution and reproduction for non-commercial purposes, provided the original is properly cited and derivative works building on this content are distributed under the same license. 